\title{
A closer look at the staging of soft tissue sarcomas in an era of personalized medicine
}

\author{
Aileen C. Johnson, Kenneth Cardona \\ Division of Surgical Oncology, Winship Cancer Institute, Emory University, Atlanta, GA, USA \\ Correspondence to: Kenneth Cardona, MD, FACS. Associate Professor of Surgery, Division of Surgical Oncology, Winship Cancer Institute, Emory \\ University Hospital Midtown, 550 Peachtree St., NE, 9th floor -- Ste 9000, Atlanta, GA 30308, USA. Email: ken.cardona@emory.edu. \\ Response to: Tseng WW, Pasquali S, Hu JS, et al. Staging systems and nomograms in soft tissue sarcoma: outcome prediction by categorization or \\ personalization? Chin Clin Oncol 2019;8 Suppl 1:S12. doi: 10.21037/cco.2019.01.05
}

Submitted Jun 06, 2019. Accepted for publication Jun 11, 2019.

doi: $10.21037 /$ cco.2019.06.01

View this article at: http://dx.doi.org/10.21037/cco.2019.06.01

We read with enthusiasm the editorials by Rick Hass and William Tseng in response to our recently published manuscript, "A novel, simplified, externally validated staging system for truncallextremity soft tissue sarcomas: An analysis of the US Sarcoma Collaborative database" (1). These editorials, along with a considerable array of recent publications, review articles, and other editorials over the past few years, represent a re-invigorated interest for this rare, and previously somewhat ignored, malignancy amongst the oncology community.

To this point, the most recent edition $\left(8^{\text {th }}\right)$ of the AJCC staging manual made considerable changes to the staging of soft tissue sarcomas, as detailed by Dr. Tseng. As described by Haas, three main goals of proposing staging systems are creating a common language, allowing comparisons of outcome parameters, and predicting outcomes (2). Successful attainment of these objectives is significantly more difficult in a rare and heterogenous disease such as soft tissue sarcoma, particularly given the numerous histotypes with distinct biologic behavior. In an era of personalized medicine, sarcoma nomograms attempt to bridge this gap and provide a more granular assessment of patient outcomes or assess risk based on specific characteristics of a patient and their disease. Thus, both staging systems and nomograms are utilized in soft tissue sarcoma with the aim of better predicting outcomes by patient characteristics; however, both approaches are also saddled with limitations.

The main criticism of staging systems in general, and our recently proposed system in particular, is the exclusion of specific histotypes, thus making them less individualized and potentially less accurate $(2,3)$. While nomograms include histotype in their outcome prediction algorithms, it is worth noting that the majority of histotypes are not accounted for in either of the two main nomograms. Only the most prevalent subtypes (5 in PERSARC and 8 in SARCULATOR) are stratified separately. Therefore, even within these nomograms, the majority of the $75+$ different sarcoma histotypes are combined into a single category of "other" $(4,5)$, much like a classical staging system. Thus, even with nomograms, the ability to predict individual outcomes is still limited for the vast majority of histotypes and is only available for a few, albeit the most common, sarcoma subtypes.

Given the current need for histotype-specific therapies and clinical trials, due to limited effective therapy for high-risk sarcoma as a whole, there is indeed a role for individualizing patient recruitment. However, when selecting patients with a wide variety of histotypes, the utilization of nomograms in trial recruitment is hindered by their limited stratification across all histotypes. An aim for the future of sarcoma treatment is a more effective therapy applicable to all high-risk soft tissue sarcomas, regardless of histotype. In essence this is a group that is identified by a well stratified staging system (1).

In this era of personalized medicine, there is absolutely a need for histotype specific analysis. Nomograms provide a platform for an individualized discussion with a patient afflicted with a common histotype, while staging systems are more easily translated across populations and institutions. Our proposed staging system aimed to simplify the AJCC 
$8^{\text {th }}$ edition classification system by eliminating unnecessary and clinically irrelevant categories and creating better discrimination between existing ones. Despite the benefits of nomograms, it is unrealistic to address all sarcoma histotypes individually in prognostic models. As we move forward, we will need to utilize a combination of staging systems and nomograms synergistically for predicting patient outcomes and clinical trial recruitment.

\section{Acknowledgments}

None.

\section{Footnote}

Conflicts of Interest: The authors have no conflicts of interest to declare.

Ethical Statement: The authors are accountable for all aspects of the work in ensuring that questions related to the accuracy or integrity of any part of the work are appropriately investigated and resolved.

Cite this article as: Johnson AC, Cardona K. A closer look at the staging of soft tissue sarcomas in an era of personalized medicine. Chin Clin Oncol 2019;8(Suppl 1):S27. doi: 10.21037/ cco.2019.06.01

\section{References}

1. Johnson AC, Ethun CG, Liu Y, et al. A novel, simplified, externally validated staging system for truncal/extremity soft tissue sarcomas: An analysis of the US Sarcoma Collaborative database. J Surg Oncol 2018;118:1135-41.

2. Haas RL, van de Sande MA. Considering sarcoma staging systems and their implications to personalized care. Chin Clin Oncol 2019. doi: 10.21037/cco.2019.01.03.

3. Tseng WW, Pasquali S, Hu JS, et al. Staging systems and nomograms in soft tissue sarcoma: outcome prediction by categorization or personalization? Chin Clin Oncol 2019;8 Suppl 1:S12.

4. van Praag VM, Rueten-Budde AJ, Jeys LM, et al. A prediction model for treatment decisions in high-grade extremity soft-tissue sarcomas: Personalised sarcoma care (PERSARC). Eur J Cancer 2017;83:313-23.

5. Callegaro D, Miceli R, Bonvalot S, et al. Development and external validation of two nomograms to predict overall survival and occurrence of distant metastases in adults after surgical resection of localised soft-tissue sarcomas of the extremities: a retrospective analysis. Lancet Oncol 2016;17:671-80. 